A preci se method for detecti on of vacuum I eakage in a pressure sensor usi ng a pul se di scharge techni que, for i ndustrial use

\begin{tabular}{|l|l|}
\hline 著者 & UEDA M SUG NO Y, KAGAWA K, MATSUl T \\
\hline $\begin{array}{l}\text { j our nal or } \\
\text { publ i cat i on t i t l e }\end{array}$ & Journal of Physi cs - London- d Appl i ed Physi cs \\
\hline vol une & 31 \\
\hline number & 12 \\
\hline page range & $1465-1470$ \\
\hline year & $1998-06$ \\
\hline URL & ht t p: //hdl . handl e. net /10098/1450 \\
\hline
\end{tabular}




\title{
A precise method for detection of vacuum leakage in a pressure sensor using a pulse discharge technique, for industrial use
}

\author{
M Ueda†, Y Sugino†, K Kagawa† and T Matsuił \\ † Faculty of Education, Fukui University, Bunkyo 3-9-1, Fukui 910-0017, Japan \\ $\ddagger$ Hokkei Industry Co Ltd, Masuizumi 3-4-20, Kanazawa 921-8025, Japan
}

\begin{abstract}
A new method for measuring the vacuum leakage of a pressure sensor proposed by the present authors has been improved so that it can be applied for industrial use. The principle of this method is based on the discharge characteristic called Pashen's law. The main improved points are reduction of fluctuation of the discharge potential and extension of the measurable pressure range. As a result, a leakage rate of $1 \times 10^{-5} \mathrm{~Pa} \mathrm{~cm} \mathrm{~s}^{-1}$ can be detected within a day. This sensitivity is comparable to that of the radio-isotope method now in practical use or even higher than that. The measurable pressure range is enlarged from $70-150 \mathrm{~Pa}$ to $70 \mathrm{~Pa}$ to a few hundreds pascals by employing $\mathrm{He}$ gas to detect the leakage.
\end{abstract}

\section{Introduction}

Vacuum leakage of a pressure sensor used in a car can often yield a serious problem even if the leakage is very small. The most sensitive and precise method now in practical use is a radio-isotope method. The method has, however, such problems as high cost and, in particular, environmental pollution. A new technique is then necessary to alleviate these problems.

In a previous paper [1], we proposed a new method for detecting vacuum leakage using a pulse discharge technique. The principle is based on the discharge characteristic called Paschen's law [2], whereby the breakdown voltage of the discharge depends on the pressure. The results suggested that the method can be used in industry. It poses, however, some problems for practical use. Two of the serious problems are the lack of sensitivity and restriction to an applicable range of pressure of the sensor. These make the method difficult to use practically in industry. In this paper, we report a new technique to solve these two main problems in order to make the method applicable in the industrial field.

\section{Improvements on the previous method}

\subsection{The previous method}

The principle and the experimental method are basically same as that reported in the previous paper [1]. Figure 1(a) shows the experimental set-up used in the pressurecontrolled simulation experiment and figure 1(b) shows the electrical circuit used to obtain the discharge characteristics of a single-shot discharge. The eight electrode pins embedded in the pressure sensor were used as an anode. They were connected to each other in order to maintain the same electrical potential. The shielding body case was used as a cathode. A high resistance (a few tens of megaohms) was used to restrict the discharge current and to avoid damaging the $I C$ circuit in the sensor due to a high-voltage discharge and an electromagnetic relay, EMR, was used to obtain a one-shot discharge with a duration of about a few microseconds (see figure 1(b)).

A simulation experiment was performed in order to obtain the discharge characteristic, that is, a relation between the discharge potential and the pressure, just like in the previous paper [1]. The experiment was performed using a pseudo-sensor, in which the shielding cap of the sensor can be removed. The removed cap is re-connected to the base of the sensor's body so that the electrical field is the same as that of the practical sensor but the pressure in the pseudo-sensor can be easily controlled.

The discharge characteristics can be used for the estimation of pressure in the sensor, that is, to provide a measurement standard of the inner pressure. The method, therefore, requires two measurements after a proper time interval $T$ in order to obtain the vacuum leakage rate.

\subsection{Improvements}

The accuracy of the estimation of pressure depends on the reproducibility of the discharge. The potential difference differs by about a few tens of volts around a mean value in 


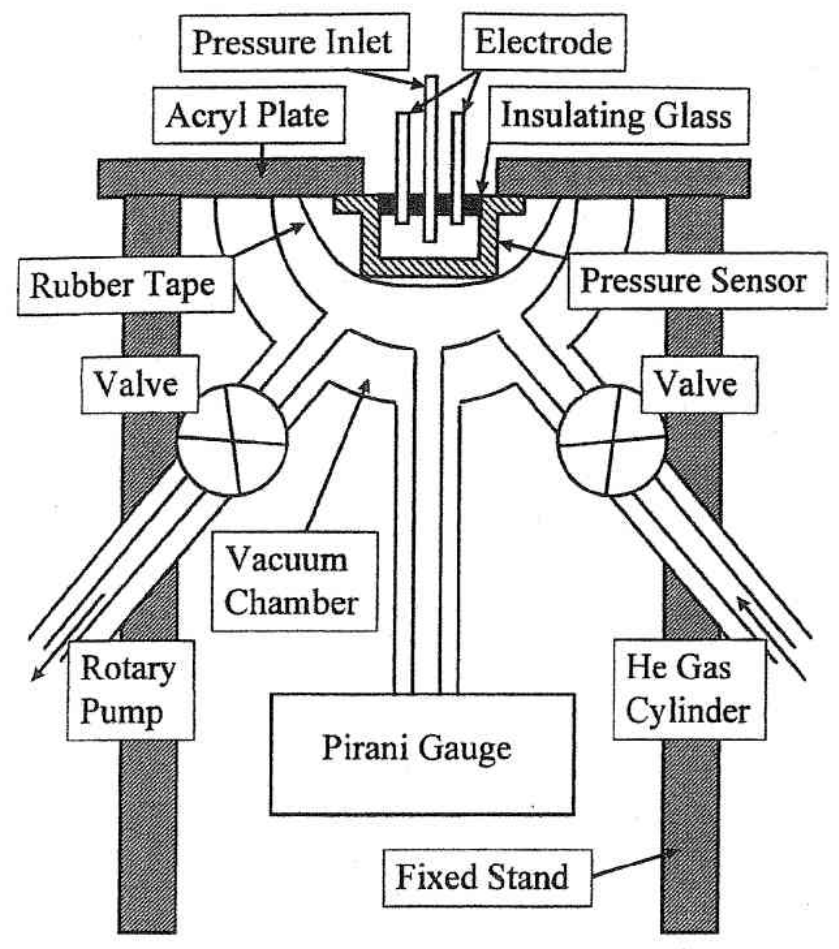

(a)

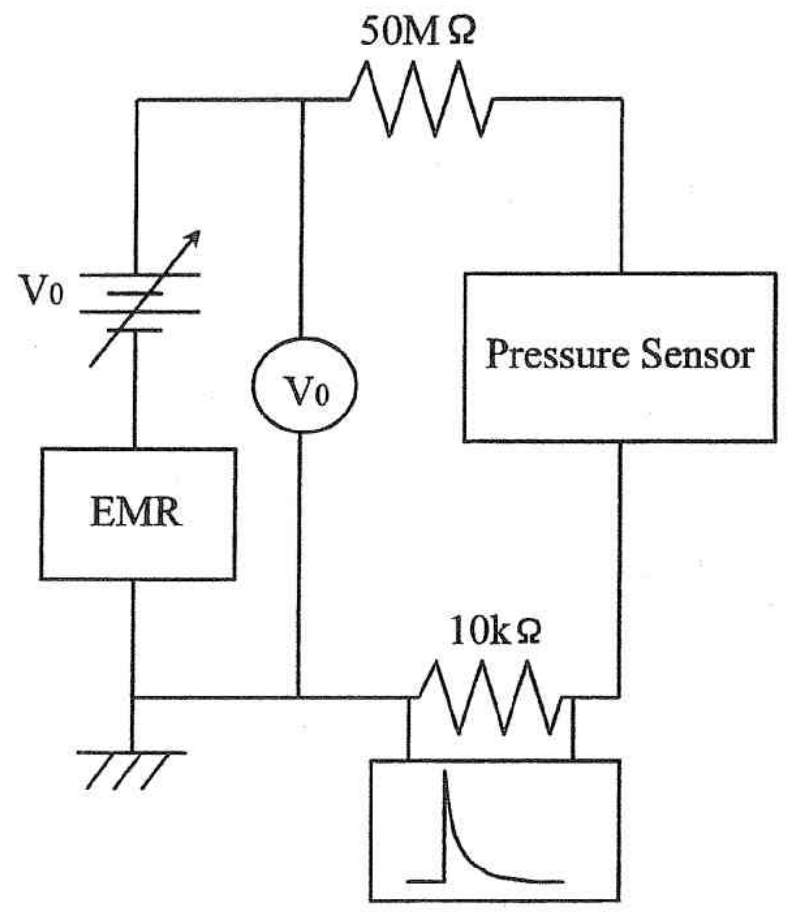

Storage Oscilloscope

(b)

Figure 1. The experimental arrangement. (a) The experimental set-up used to obtain the pressure-controlled simulation experiment and (b) the electrical circuit for the single-shot discharge.

each measurement even if the pressure could be maintained constant [1]. The difference, namely, fluctuation, of the discharge potential means that one requires a long time interval $T$, for example a week, in order to detect a maximum allowable vacuum leakage rate, in this case $1 \times 10^{-5} \mathrm{~Pa} \mathrm{~cm}^{3} \mathrm{~s}^{-1}$. This was the main disadvantage

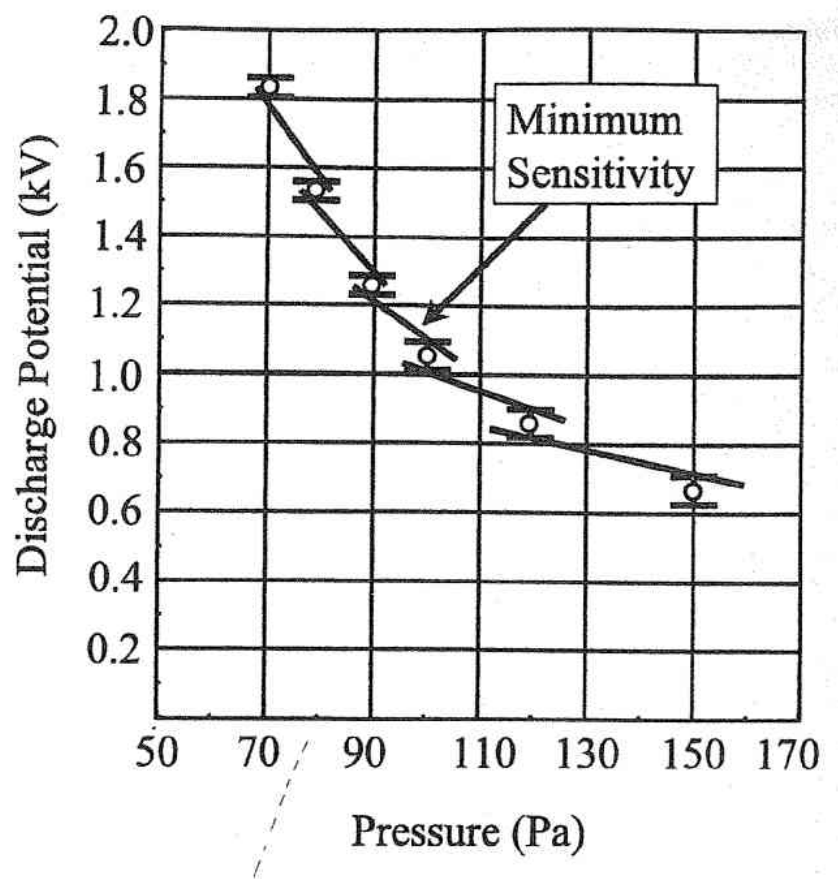

Figure 2. Discharge characteristics for a pseudo-sensor sample. Ten measurements were performed for each pressure.

and prevented the method from being practically used in the car industry.

In this study, we have made three improvements to reduce the fluctuation of the discharge potential. Firstly, electric conductive paste was used to keep a good electric connection between the eight electrical pins leading out and the high-voltage power line. Secondly, prior to an inspection discharge, a pre-discharge was carried out to flush the surface of the pressure sensor. Thirdly, the optimum speed of raising the potential from zero to the discharge potential was sought and found to be $30 \mathrm{~V} \mathrm{~s}^{-1}$.

Another main disadvantage was that the method can only be applied effectively within a narrow range of inner pressure, 70-150 $\mathrm{Pa}$. This also restricts the method for applications in the industrial field because the usual inner pressure range is $70-300 \mathrm{~Pa}$ due to many factors involved in making the practical sensor. That is, our method is quite useless above $150 \mathrm{~Pa}$, where it has little sensitivity, as is discussed in the next section. We have, however, found a new technique using $\mathrm{He}$ gas to solve this problem. Addition of a small amount of $\mathrm{He}$ gas has a large effect on the discharge. It makes the method useful in the pressure range above $150 \mathrm{~Pa}$.

\section{Results and discussion}

A simulation experiment was performed to obtain discharge characteristics using three pseudo-sensors, A, B and C. Figure 2 shows an example of the discharge characteristics for sample $\mathrm{A}$ in the pressure range $70-150 \mathrm{~Pa}$. This is the well-known Paschen law [2], which has been obtained by using an ordinary discharge. The fluctuations of the discharge potential, namely the differences between maximum and minimum values, in ten measurements were 
Table 1. Minimum sensitivities $S$ defined by $S_{\min }=\Delta V_{\min } / \Delta P$, where $\Delta V_{\min }$ is the minimum difference between the discharge potentials in each pressure range and $\Delta P$ is the difference in pressure.

\begin{tabular}{llllll}
\hline & \multicolumn{5}{c}{ Pressure (Pa) } \\
\cline { 2 - 6 } Sample & $70-80$ & $80-90$ & $90-100$ & $100-120$ & $120-150$ \\
\hline A & & 25.0 & 21.5 & 10.8 & 6.9 \\
B & 27.1 & 23.9 & 14.2 & 6.0 & 4.5 \\
C & 40.8 & 21.5 & 10.4 & 6.1 & 4.5 \\
\hline
\end{tabular}

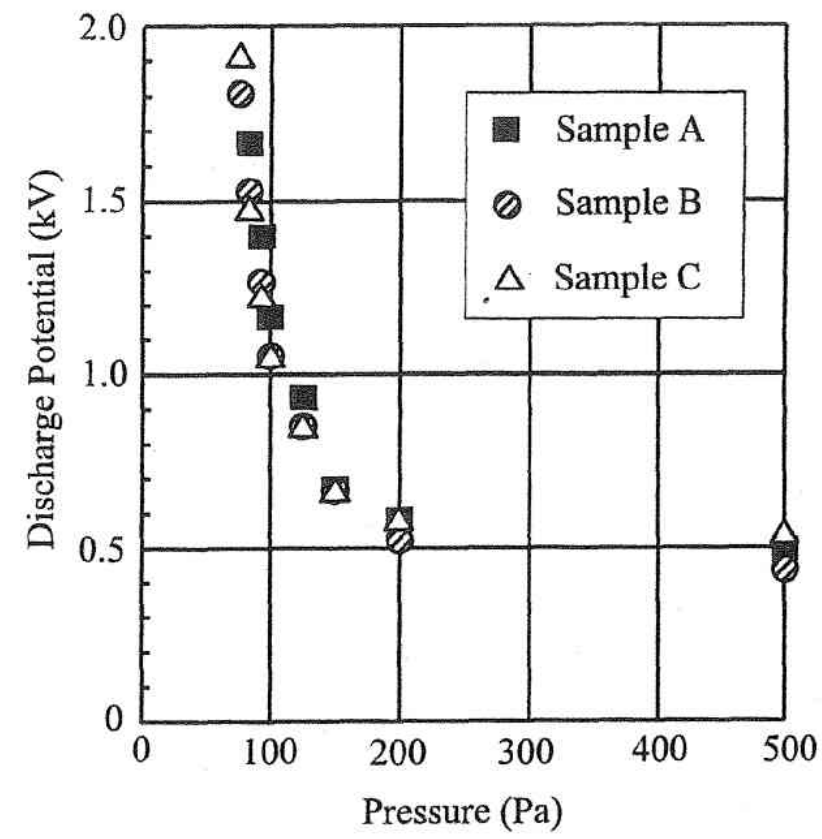

Figure 3. Discharge characteristics for three typical sensor samples. The value at each pressure is the mean value of ten measurements.

all below $70 \mathrm{~V}$ at any given pressure. The other two samples, B and C, have also almost the same fluctuation as that of sample $\mathrm{A}$ in the pressure range $70-150 \mathrm{~Pa}$. These fluctuation are considerably reduced, to about a half the original value of the previous one without the improvement.

It is generally believed that the discharge potential fluctuates greatly and it is essentially difficult to use this method for estimation of pressure with high precision. It is, however, supposed that, under limited conditions, the fluctuation of the discharge potential can be decreased to a considerable extent. In this experimental case, the discharge is supposed to occur on the surface of glass which is used as an insulator between the electrode pins and the base metal of the pressure sensor. This was proved by the fact that the discharge potential is almost the same even when the shielding cap was removed in the simulation experiment. The method has thus been proved to be suitable for use in industry.

Figure 3 shows the discharge characteristics for three pseudo-sensor samples in the pressure range 70-500 $\mathrm{Pa}$. The discharge potential is a mean value in figure 3 . These characteristics almost coincide with each other. There is, however, a little difference for each sample. This causes a problem regarding which characteristic curve is used when the pressure of a practical sensor is estimated from the measured discharge potential.

It is useful to define a sensitivity $S$ as follows for evaluation of the method in practical use:

$$
S=\Delta V / \Delta p
$$

where $\Delta V$ is a small change of the discharge potential due to a small change in pressure, $\Delta p$. The sensitivity depends strongly on the pressure, as can be seen in figures 2 and 3 . A large $S$ means a high sensitivity in estimating the pressure from the discharge potential. It is natural to use a mean sensitivity, mean $S$, which is obtained from the averaged discharge potential at each pressure. In the previous paper, we used the mean sensitivity. It may, however, be more useful to use the minimum sensitivity to find out for certain the maximum allowable leakage rate of the sensor, $R_{m}$. That is, this never misses leakage rates larger than $R_{m}$ and is essential for industrial use from the viewpoint of safety planning. The minimum sensitivity, $S_{\min }$, can be obtained from the straight line shown in figure 2 . The standard $R_{m}$ now used in the car industry is $1 \times 10^{-5} \mathrm{~Pa} \mathrm{~cm}^{3} \mathrm{~s}^{-1}$, which is obtained from consideration of a car life time of about 15 years.

Table 1 shows the minimum sensitivities for three pseudo-sensor samples, A, B and C, one of which is obtained from figure 2. The blank column for sample A means that the discharge did not take place even at $2000 \mathrm{~V}$, which is the maximum supply voltage in this experiment. The error in pressure due to the fluctuation of the discharge potential can be determined by the values in table 1 .

Table 2 shows the discharge potential of the practical pressure sensors. Two kinds of sensor were used: one has a high discharge potential, $1100-1500 \mathrm{~V}$, which means that the inner pressure of the sample is estimated to be relatively low, 80-100 Torr, as can be seen in figure 3 , whereas the other has a low potential, $500-600 \mathrm{~V}$, namely a high inner pressure of around 150-200 Torr.

Ten measurements were performed for each sensor. The maximum fluctuations of the discharge potential, $V_{p-p}$, were all smaller than $25 \mathrm{~V}$ for this kind of low-pressure type. The fluctuation was, however, about $40 \mathrm{~V}$ for the high-pressure type. A rather small fluctuation can thus be obtained by implementing the improvements mentioned in section 2.2. The fluctuation causes a presumed error in pressure. The reduction of this fluctuation is one of the most important factors for this method to be effectively used in the industrial field. That is, the error can be used as a standard for a clear distinction of vacuum leakage from error in pressure. The presumed error in pressure depends also on the sensitivity mentioned above. For example, the error in pressure can be calculated to be $2.4 \mathrm{~Pa}(=25 / 10.4)$ for the fluctuation potential of $25 \mathrm{~V}$ and the minimum sensitivity of $10.4 \mathrm{~V} \mathrm{~Pa}^{-1}$. The presumed error in pressure, finally, determines the accuracy of this method, in other words, determines the shortest time required in order to detect the allowable maximum leakage rate. The required time can, thus, be determined from the presumed error in pressure due to the potential fluctuation, the minimum sensitivity, the allowable maximum leakage rate and the sensor's volume. 
Table 2. Some examples of discharge potentials observed in 16 practical sensors. The fluctuation of the discharge potential is shown as the peak-peak $(p-p)$ value. Two kinds of sensors were used; one has a high discharge potential and the other has a low discharge potential.

\begin{tabular}{|c|c|c|c|c|c|c|c|c|c|c|c|c|}
\hline \multirow{2}{*}{$\begin{array}{l}\text { Sensor } \\
\text { number }\end{array}$} & \multicolumn{12}{|c|}{ Discharge potential (V) } \\
\hline & 1 st & 2nd & 3rd & 4th & 5th & 6th & 7th & 8th & 9th & 10th & $V_{\text {ave }}$ & $V_{p-p}$ \\
\hline 1 & 1492 & 1484 & 1476 & 1468 & 1468 & 1468 & 1484 & 1492 & 1492 & 1468 & 1479 & 24 \\
\hline 2 & 974 & 990 & 974 & 974 & 974 & 991 & 982 & 982 & 990 & 990 & 982 & 17 \\
\hline 3 & 1229 & 1229 & 1214 & 1229 & 1222 & 1229 & 1237 & 1237 & 1229 & 1237 & 1229 & 23 \\
\hline 4 & 1213 & 1214 & 1213 & 1198 & 1198 & 1214 & 1222 & 1214 & 1214 & 1214 & 1211 & 24 \\
\hline 5 & 1477 & 1488 & 1488 & 1489 & 1489 & 1477 & 1500 & 1489 & 1489 & 1500 & 1489 & 23 \\
\hline 6 & 1275 & 1299 & 1299 & 1299 & 1299 & 1275 & 1287 & 1299 & 1299 & 1299 & 1293 & 24 \\
\hline 7 & 1072 & 1049 & 1072 & 1072 & 1049 & 1049 & 1072 & 1061 & 1061 & 1072 & 1063 & 23 \\
\hline 8 & 1097 & 1084 & 1109 & 1097 & 1109 & 1109 & 1109 & 1109 & 1109 & 1109 & 1104 & 25 \\
\hline 9 & 544 & 528 & 544 & 502 & 544 & 528 & 502 & 502 & 502 & 512 & 521 & 42 \\
\hline 10 & 568 & 545 & 576 & 545 & 553 & 584 & 568 & 584 & 568 & 545 & 564 & 39 \\
\hline 11 & 470 & 470 & 430 & 454 & 462 & 430 & 430 & 430 & 430 & 446 & 445 & 40 \\
\hline 12 & 576 & 576 & 544 & 576 & 536 & 536 & 536 & 568 & 576 & 560 & 558 & 40 \\
\hline 13 & 536 & 544 & 544 & 536 & 536 & 528 & 536 & 536 & 544 & 544 & 538 & 16 \\
\hline 14 & 624 & 616 & 624 & 656 & 656 & 624 & 616 & 616 & 624 & 616 & 627 & 40 \\
\hline 15 & 608 & 640 & 632 & 608 & 608 & 608 & 640 & 624 & 608 & 624 & 620 & 32 \\
\hline 16 & 656 & 656 & 632 & 656 & 616 & 648 & 632 & 616 & 616 & 624 & 635 & 40 \\
\hline
\end{tabular}

Table 3. The sensitivity of this method in terms of the estimated maximum error in pressure caused by a fluctuation in potential of $25 \mathrm{~V}$.

\begin{tabular}{llllll}
\hline & \multicolumn{5}{c}{ Pressure $(\mathrm{Pa})$} \\
\cline { 2 - 6 } Sample & $70-80$ & $80-90$ & $90-100$ & $100-120$ & $120-150$ \\
\hline $\mathrm{A}$ & & 1.00 & 1.16 & 2.32 & 3.62 \\
$\mathrm{~B}$ & 0.92 & 1.05 & 1.76 & 4.17 & 5.56 \\
$\mathrm{C}$ & 0.61 & 1.16 & 2.40 & 4.10 & 5.56 \\
\hline
\end{tabular}

Table 4. The sensitivity of this method in terms of the minimum number of days required in order to detect the maximum allowable leakage rate of $1 \times 10^{-5} \mathrm{~Pa} \mathrm{~cm}^{3} \mathrm{~s}^{-1}$ for a practical sensor.

\begin{tabular}{llllll}
\hline & \multicolumn{5}{c}{ Pressure (Pa) } \\
\cline { 2 - 6 } Sample & $70-80$ & $80-90$ & $90-100$ & $100-120$ & $120-150$ \\
\hline A & & 0.58 & 0.68 & 1.34 & 2.10 \\
B & 0.53 & 0.61 & 1.02 & 2.41 & 3.22 \\
C & 0.35 & 0.67 & 1.39 & 2.37 & 3.22 \\
\hline
\end{tabular}

The increase in pressure can be calculated to be $1.7 \mathrm{~Pa}$ per day if the sensor leaks with the maximum leakage rate of $R_{m}=1 \times 10^{-5} \mathrm{~Pa} \mathrm{~cm} \mathrm{cs}^{-1}$ and the sensor's volume is $0.5 \mathrm{~cm}^{3}$. For example, the time required can, then, be calculated to be $1.39(=2.4 / 1.7)$ days for the presumed error in pressure of $2.4 \mathrm{~Pa}$.

Table 3 shows the presumed error in pressure and table 4 shows the required time in days for three samples, $\mathrm{A}, \mathrm{B}$ and $\mathrm{C}$, and various pressure ranges. These are used for the measurement standard of this method.

The values, however, differ slightly from each other. We, may, therefore, use a smaller sensitivity and then a longer detecting time in order to take a safe measurement.
Table 5. The effect of He gas on the discharge characteristics.

\begin{tabular}{llllll}
\hline & \multicolumn{2}{c}{ Air (Pa) } & & \multicolumn{2}{c}{ Air + He gas $(\mathrm{Pa})$} \\
\cline { 2 - 3 } \cline { 5 - 6 } Discharge & 150 & 190 & & $150+68$ & $190+28$ \\
\hline First & 649 & 644 & & 1196 & 785 \\
Second & 623 & 574 & & 1172 & 825 \\
\hline
\end{tabular}

From the practical point of view, the time interval $T$ between the two measurements to obtain the vacuum leakage rate should be less 3 days. Therefore, it is concluded from tables 3 and 4 that the method can only be useful for the pressure range $70-150 \mathrm{~Pa}$; an example of this is sensors $1-8$ in table 2 .

The sensitivity decreases abruptly when the inner pressure is over $150 \mathrm{~Pa}$. That is, the method cannot be applied to a sensor with an inner pressure above $150 \mathrm{~Pa}$; an example of this pressure range is sensors 9-16 in table 2. The lack of sensitivity is an unavoidable disadvantage of this method. We have, however, found a new phenomenon allowing us to solve this problem. Namely, the sensitivity can be increased by employing He gas, instead of air, as the surrounding gas of the sensor.

Table 5 shows the relation between the discharge potential and the total inner pressure for a sensor filled with air and He gas. As shown in figure 3, the discharge potential surely decreases as the inner pressure increases in the pressure range 70-500 $\mathrm{Pa}$. It was, however, found that addition of $\mathrm{He}$ gas increases the discharge potential and the amount of the gas determines the increase in potential. We can then devise a sensor with a pinhole for gas flow. For this purpose, sensors with a high pressure, above $150 \mathrm{~Pa}$, namely a low discharge potential, must be set in a chamber filled with only $\mathrm{He}$ gas for a few days. A detailed experiment and a theoretical analysis will be required for the determination of the leakage rate. 


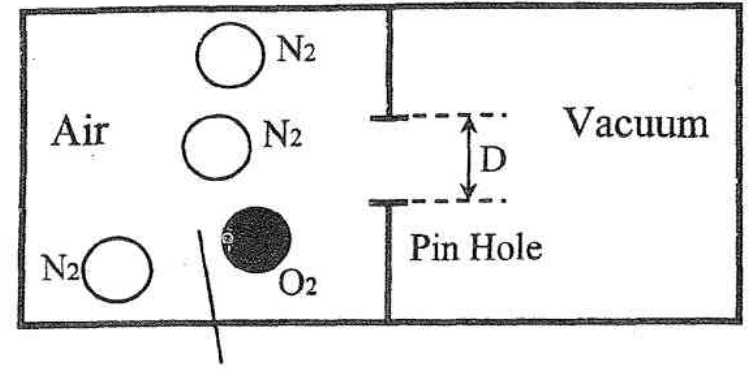

Atmosphere

(a)

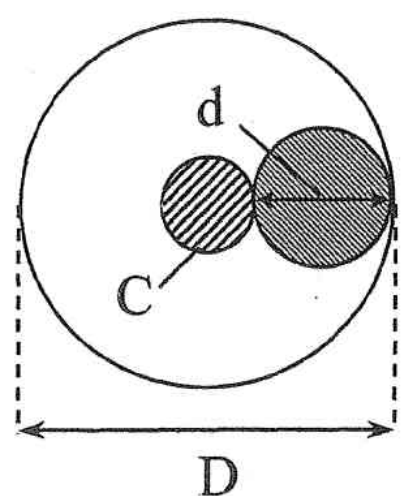

(b)

Figure 4. A schematic diagram of the gas leak: (a) a model of the pinhole and (b) the concept of gas leakage.

Only a theoretical analysis for the leakage rate of each gas was performed for the estimation of the amount of each gas that leaked into the sensor. Figure 4(a) shows a conceptual expression for the pinhole and figure 4 (b) is for gas leakage. Two chambers are isolated by a wall with a small pinhole; one is maintained at vacuum and the other at atmospheric pressure.

The basis of the theory is to calculate the dimension of the pinhole for a given leakage rate and the amount of gas being leaked through the pinhole. The amount of gas leaked, $\Delta N$, can be expressed, from a consideration of thermo-statistical mechanics [3], as

$$
\Delta N=\frac{1}{4} n S V
$$

where $n$ is the number density of gas molecules, $S$ is the cross sectional area of the pinhole and $V$ is the thermal velocity. $S$ can be calculated to be

$$
S=0.774 \times 10^{-18} \mathrm{~m}^{2}
$$

assuming a maximum allowable leakage rate of $R_{m}=$ $1 \times 10^{-5} \mathrm{~Pa} \mathrm{~cm}^{3} \mathrm{~s}^{-1}$. If the area is assumed to be a disc, the diameter $D$ can be calculated to be

$$
D=1 \mathrm{~nm} \text {. }
$$

Molecular diameters $d$ of $\mathrm{He}, \mathrm{N}_{2}$ and $\mathrm{Kr}$ are given as follows [4]:

$$
\begin{gathered}
d_{H e}=0.218 \mathrm{~nm} \quad d_{N_{2}}=0.375 \mathrm{~nm} \\
d_{K r}=0.416 \mathrm{~nm} .
\end{gathered}
$$

$\mathrm{N}_{2}$ gas is considered as air in this case. The effective cross sectional area $C$ by which each molecule goes past the pinhole without colliding with a wall, therefore, can be obtained as

$$
\begin{gathered}
C_{H e}=7.5 \pi \times 10^{-20} \mathrm{~m}^{2} \quad C_{N_{2}}=1.4 \pi \times 10^{-20} \mathrm{~m}^{2} \\
C_{K r}=0.52 \pi \times 10^{-20} \mathrm{~m}^{2} .
\end{gathered}
$$

The leakage rate $R$ is directly proportional to this effective cross sectional area and the mean thermal velocity $V$ which can be calculated to be $V_{H e}=1.367 \mathrm{~km} \mathrm{~s}^{-1}$, $V_{N_{2}}=0.516 \mathrm{~km} \mathrm{~s}^{-1}$ and $V_{K r}=0.299 \mathrm{~km} \mathrm{~s}^{-1}$ assuming a room temperature of $T=300 \mathrm{~K}$. We can, then, obtain the relation of the leakage rates for each gas, as follows:

$$
R_{H_{e}}: R_{N_{2}}: R_{K r}=14.5: 1.0: 0.22 \text {. }
$$

The result shown above was obtained by assuming that the pinhole is just a disc in shape. The actual shape of the pinhole may be an ellipse and the ratio may become larger than that of equation (6). Therefore, it is assumed that the inner pressure due to leakage of gas into the sensor increases faster for $\mathrm{He}$ than it does for air by a factor of about a few tens. As a result, the increase in pressure during a day is expected to be about 30$50 \mathrm{~Pa}\left(=1.7 \times 1 \times R_{\mathrm{He}} / R_{N_{2}}\right.$ ) for the maximum allowable leakage rate of $R_{m}=1 \times 10^{-5} \mathrm{~Pa} \mathrm{~cm}^{3} \mathrm{~s}^{-1}$. This amount of gas pressure increment would sufficiently increase the discharge potential, as can be seen from table 5. This makes the method effective even in the pressure range above $150 \mathrm{~Pa}$. The reason why the addition of $\mathrm{He}$ increases the discharge potential remarkably compared with the case for air may be due to the fact that the ionic potential of $\mathrm{He}(24.58 \mathrm{eV})$ is far higher than that of $\mathrm{N}_{2}$ $(15.58 \mathrm{eV})$.

The most sensitive method now used for the detection of air leakage is a radio-isotope method. It requires about 2 days to detect the maximum allowable leakage rate of $R_{m}=1 \times 10^{-5} \mathrm{~Pa} \mathrm{~cm} \mathrm{~cm}^{-1}$ clearly. The method may, however, have a problem from the consideration mentioned above, namely that air leaks although $\mathrm{Kr}$ does not leak when the cross section of the pinhole is not a disc but rather a hole with the shape of a long and narrow ellipse.

\section{Conclusion}

The new method to detect the vacuum leakage of a pressure sensor previously reported has been improved further in order for it to be applied in the industrial field. The improved method has the advantages over the previous method that

(i) the fluctuation of the discharge potential is decreased to about a half that of the previous result by application of some techniques and

(ii) the applicable pressure range is extended from $70-150 \mathrm{~Pa}$ to $70 \mathrm{~Pa}$ to a few hundred pascals by employing $\mathrm{He}$ as the surrounding gas to detect the leakage. 
It can be concluded that the efficiency of this method is equal to or higher than that of the radio-isotope method now in practical use.

\section{Acknowledgment}

Technical support throughout the present work was provided by Mr T Inaki of Fukui University.

\section{References}

[1] Ueda M, Kagawa K, Sugino Y, Moriya K, Chen J and Matsui T 1997 J. Phys. D: Appl. Phys. 30 703-7

[2] Brown S C 1996 Introduction to Electrical Discharge in Gases (New York: Wiley) p 3

[3] Reif F 1965 Fundamentals of Statistical and Theoretical Physics (New York: McGraw-Hill) p 269

[4] Kennard E H 1938 Kinetic Theory of Gases (New York: McGraw-Hill) p 149 